\title{
Review Article \\ Interactive Digital Serious Games for the Assessment, Rehabilitation, and Prediction of Dementia
}

\author{
Sayed Kazmi, ${ }^{1}$ Hassan Ugail, ${ }^{1}$ Valerie Lesk, ${ }^{2}$ and Ian Palmer ${ }^{3}$ \\ ${ }^{1}$ Centre for Visual Computing, University of Bradford, Bradford, West Yorkshire BD7 1DP, UK \\ ${ }^{2}$ Division of Psychology, University of Bradford, Bradford, West Yorkshire BD7 1DP, UK \\ ${ }^{3}$ School of Media, Design \& Technology, University of Bradford, Bradford, West Yorkshire BD7 1DP, UK
}

Correspondence should be addressed to Sayed Kazmi; sayedkazmi@hotmail.com

Received 13 June 2014; Revised 30 October 2014; Accepted 3 November 2014; Published 24 November 2014

Academic Editor: Soraia Raupp Musse

Copyright (C) 2014 Sayed Kazmi et al. This is an open access article distributed under the Creative Commons Attribution License, which permits unrestricted use, distribution, and reproduction in any medium, provided the original work is properly cited.

\begin{abstract}
Dementia is a serious, progressive, and often debilitating illness with no known cure, having a severe adverse effect on memory, behaviour, reasoning, and communication. A comprehensive review of current refereed research material in the use of games in this area is scarce and suffers from being orientated towards commercially available games or derivatives such as "Dr. Kawashima's brain training." There is much lesser concern for bespoke research grade alternatives. This review will attempt to assess the current state of the art in research orientated games for dementia, importantly identifying systems capable of prediction before the onset of the disease. It can be ascertained from the literature reviewed that there are clearly a large number of interactive computer game based mechanisms used for dementia. However, these are each highly intrusive in terms of affecting normal living and the patient is aware of being tested; furthermore their long-term or real benefits are unknown as is their effect over conventional tests. It is important to predict cognitive impairment at a stage early enough to maximise benefit from treatment and therapeutic intervention. Considering the availability, use, and increasing power of modern mobile smartphones, it is logically plausible to explore this platform for dementia healthcare.
\end{abstract}

\section{Introduction}

Dementia is most commonly a neurodegenerative disease with no cure [1]. It usually occurs in the elderly causing deficits in memory, behaviour, reasoning, and communication [2] and is devastating for the lives of both the sufferers and their families [3].

According to a recent WHO report, there are currently 35.6 million sufferers with dementia globally, with 7.7 million new cases each year which suggests that there is a new case every four seconds [4]. In the United Kingdom, one in three people over 65 will develop dementia, and it already affects the lives of 800,000 sufferers nationally, expected to rise by over 100 percent to 1.7 million by 2051 . These numbers are made all the more astonishing by the fact that they only account for the $43 \%$ of UK sufferers that have actually been diagnosed with the illness, with a large number of cases still going undetected [5]. Furthermore, dementia poses a critical and substantial threat to the world's health systems, currently costing over 375 billion pounds to service annually [6], and is responsible for costing 23 billion pounds to UK society alone [7]. Additionally, this is expected to increase rapidly at a rate that outstrips the growth in actual cases of sufferers of the disease. This has resulted in the UN branding this phenomenon a public health priority requiring urgent attention [8]. Diagnosis of dementia involves neuroimaging, blood testing, and neuropsychological assessment. There is much drive to diagnose dementia as early as possible before degeneration occurs to an extreme level [9].

Interactive computer based games presently enjoy a high level of research activity in both diagnostic assessment and rehabilitative care for dementia patients [10]. The rationale behind this is to increase efficiency of the assessment and diagnostic process of dementia using a number of innovative techniques.

Despite this current refereed research material is scarce and suffers from being orientated towards a commercial game or a derivative of this, with a lesser concern for academic 
level alternatives. In contrast this review will initially assess the current state of the art in using research grade games in the arena of dementia care but will importantly ascertain what serious gaming mechanisms exist that are able to predict the onset of dementia before significant cognitive impairment occurs. McCallum and Boletsis in their review [11] have stated that although the use of electronic games in the area of dementia healthcare has received a significant level of research attention, it is still worthy of investigation. It is evident from the findings of [11] that there are currently several video games that have been developed to concentrate on various aspects and stages of the diseases progression and have shown a positive effect on those suffering from dementia. The games they have reviewed are principally designed to delay the continued deterioration of cognitive ability. In addition to this, the games are thought to enhance the quality of life for sufferers in a number of ways. Firstly, they can improve the degree of independence for the participant; Wiifit [12] has enabled users to exercise freely and without supervision. Secondly, they can help improve social interaction; MinWii [13] was able to help develop positive interactions with caregivers, whilst Lumosity [14] helped successfully engage participants with a mild cognitive impairment (MCI) in regular social activity. Thirdly, games have been proven to reduce stress levels and generate a calm mental state; MinWii [13], BigBrain Academy [15], and WiiSports [16] have each demonstrated a positive impact on depression, general behaviour, and disposition, whilst the eMotiva game collection [1] has demonstrated an ability to promote a relaxed state of mind in dementia patients.

However the McCallum and Boletsis literature review [1] lacks detail regarding many key innovations currently present in the area such as the comprehensive screening test as devised by [17]. Their work mainly focuses on adapted derivatives of commercially available games that are opposed to those that employ more academic grade alternatives, a highly important new innovation in dementia care. A key case in point is the visuospatial study for Alzheimer's disease diagnosis as developed by [18].

\section{Materials and Methods}

The remainder of this review will concentrate on specific types of games that have been developed for dementia healthcare (Tables 1, 2, and 3 provide an overview of each according to purpose), particularly academic level serious games. Key innovations in this area will be discussed, which have had results published in well-respected and established refereed technical journals such as those from the Institute of Electrical and Electronics Engineers (IEEE), Association for Computing Machinery (ACM), and those published by Springer science, after extensive online user search conducted using each journals respective digital repository and Google Scholar.

The review itself is separated into a series of broad categories that best represent the different primary purposes of each gaming system studied, and these are the following.
2.1. Assessment Mechanisms. These are serious games that aim to provide information that is useful for diagnosis. These games apply standard neuropsychological assessments such as the Montreal Cognitive Assessment (MoCA) and the MiniMental State Examination (MMSE) into the digital gaming domain.

2.2. Rehabilitative Mechanisms. These are serious games designed to significantly improve users cognitive function.

2.3. Predictive Mechanisms. These are serious games that have the capacity to predict the onset of dementia prior to the patient being given a formal diagnosis.

We will now review each one in turn.

\section{Results}

3.1. Assessment Mechanisms. Jimison et al. [19] have designed a system based upon the FreeCell card game that is designed to detect cognitive impairment in older users noninvasively over a sustained period of time. Their system utilises a performance metric for test subjects that is derived from a combination of measures such as the number of moves required to complete the level, average user efficiency (whether each move brings the game closer or further from completion), game outcome (win/loss), and time taken to complete the game.

This metric is ultimately compared against the results of a brief psychological assessment for each patient to test the validity of the methodology regarding its ability to correctly diagnose cognitive decline. The study has shown that it is possible to detect $\mathrm{MCI}$ as a result of monitoring user game performance and is signified by a higher sensitivity to difficulty and score variability for MCI patients in contrast to normal players.

Testing involved a 3-week observation period using 9 test subjects that were selected according to their interest and experience in using computer games.

Reference [20] has developed a system that has created a limited computerised version of the MoCA. The MoCA is a neuropsychological test that investigates a number of cognitive functions such as short-term memory, visuospatial ability, executive function, attention, concentration, working memory, language, and orientation [21]. The computerised version utilises electronic cubes that participants need to physically manipulate in order to provide a response. Ultimately presenting a series of seven games that each relate to a specific area within the MoCA test specifically; matching, number arranging, counting, calculating, classification, memory and orientation.

The game reviewed provides a simple assessment mechanism for cognitive deficit; however it is also essential to provide an overview of systems that are designed to maintain or enhance a user's cognitive function.

3.2. Rehabilitative Mechanisms. Imbeault et al. [22] have designed a serious game with the attempt to provide rehabilitation of cognitive function in Alzheimer's sufferers. The game 


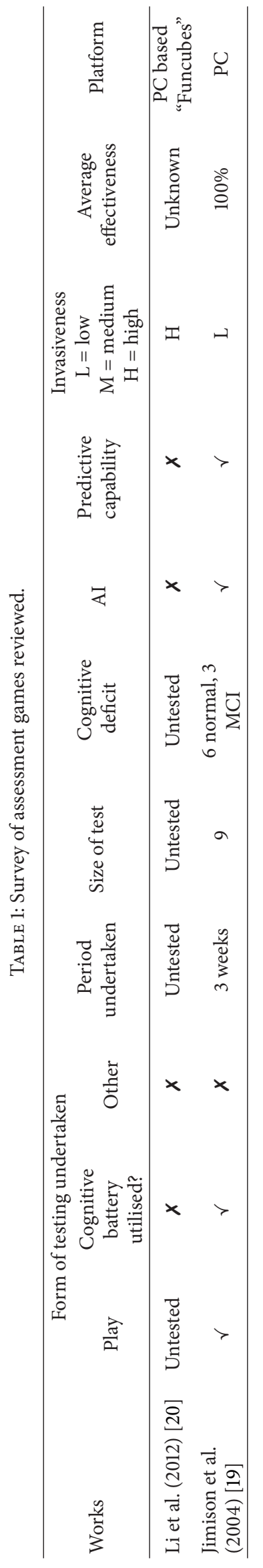




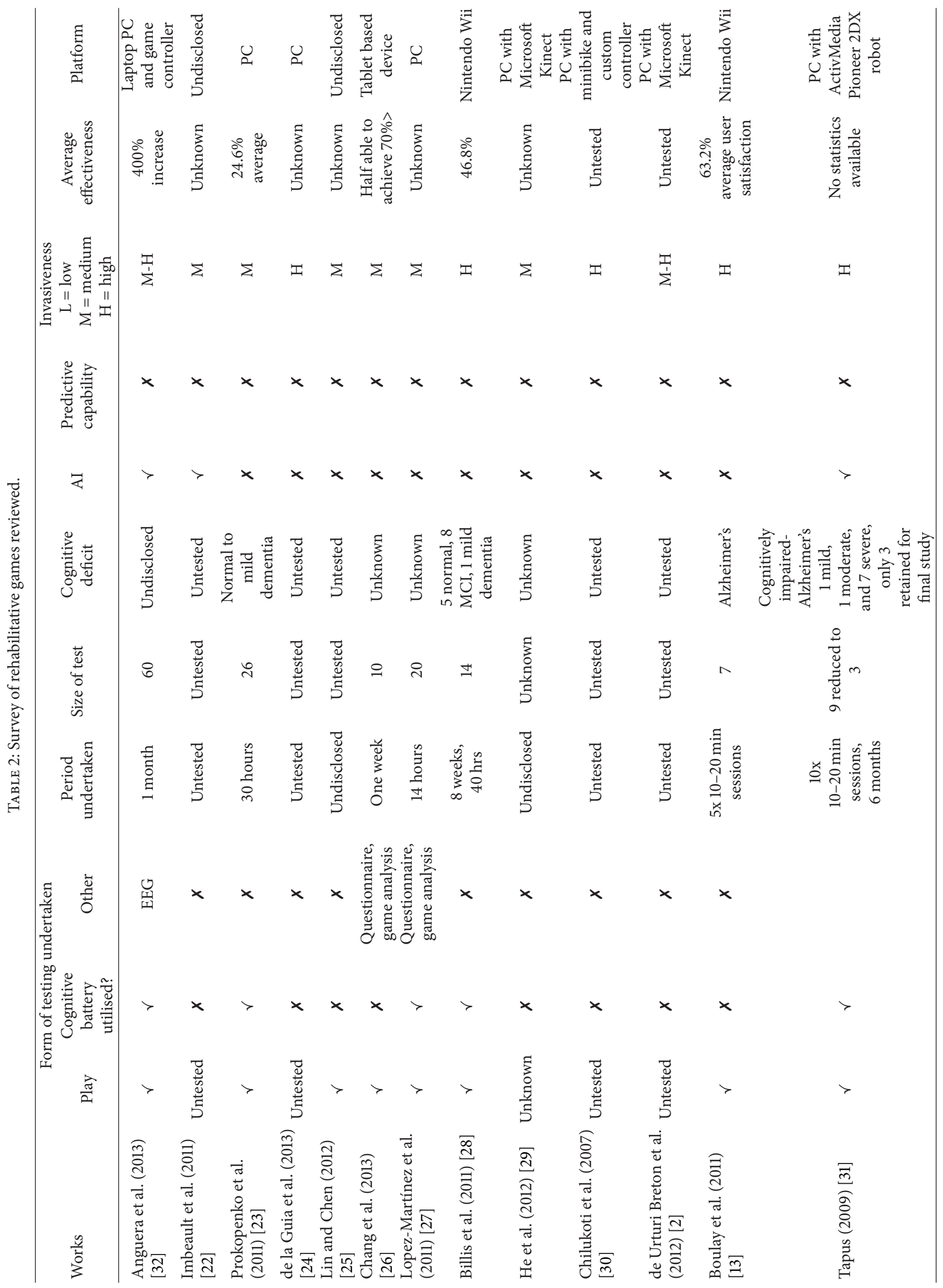




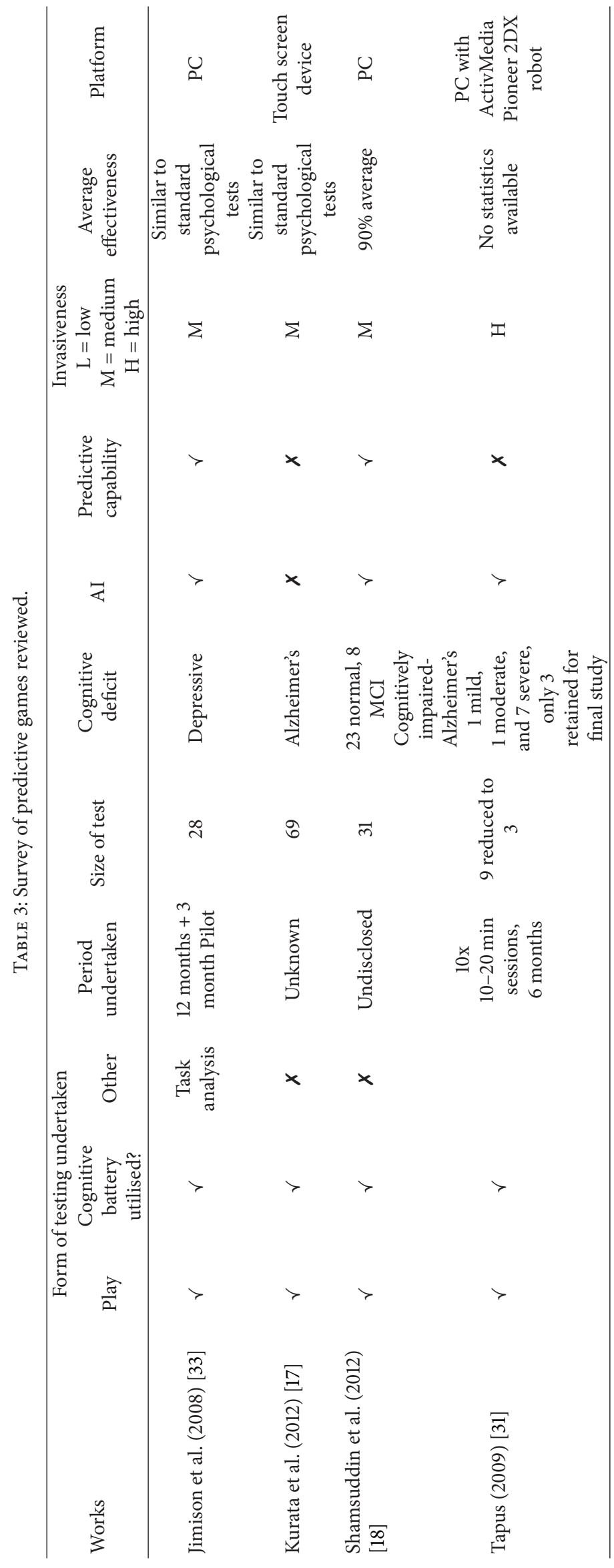


itself is a two-dimensional digitised version of the naturalistic action test. This is effectively a standard cognitive assessment that gauges how well patients can undertake a series of normal everyday activities expected of a typical person on a regular basis, for example, preparing toast and coffee for breakfast.

Prokopenko et al. [23] have designed a system that uses a series of games to test and exercise a variety of cognitive functions, namely,

(1) attention training using Schulte's tables, where the user needs to recognise and select a highlighted number from an onscreen matrix of numerical values;

(2) training visual/spatial comprehension, where the user needs to identify a shape that is partially obscured;

(3) training of visual/spatial memory, which uses a grid of images that mimic cards that are momentarily upturned on selection that the user needs to match.

Their system utilised 40 randomised test subjects in the restorative period of a stroke resulting in mild cognitive impairment and on a stationary course of neurorehabilitation, ultimately using a group of 14 control test subjects versus a basic group of 26 test subjects.

de la Guia et al. [24] have designed a system to improve the cognitive abilities of older patients with Alzheimer's using a low technology platform to remedy potential low computer literacy amongst test participants, allowing them to use a digital system effectively.

Their system functions by having participants observe on screen prompts and moving a mobile phone device across a physical playing board to the correct corresponding image. It employs the use of near field communication (NFC) technology that allows data to be communicated across two devices through touch, which is housed within the phone and an associated aerial in each image on the board.

Lin and Chen [25] have designed a game that imitates orchid planting in a virtual environment in order to increase relaxation and mental exercise for older users with cognitive impairment.

Chang et al. [26] have developed a system based on reminiscence therapy that is used to assess and improve a patient's memory and calculation ability in order to reduce the onset of cognitive impairment. Their system presents a digital shop where the patient must use food stamps (a form of currency in China during the 1960s that may resonate with older patients) to trade for specific items available for purchase.

Lopez-Martínez et al. [27] have designed a game that uses a computer based training platform to assist planning and organisation skills as well as enhancing focus and motivation in sufferers of cognitive impairment. The basic premise of the game builds on a similar concept of trading as outlined by the works of [26] but in this case provides a higher degree of emphasis on the rehabilitation of cognitive goal planning. Gameplay is focused on the user having to purchase a number of gifts for a fictitious relative while keeping to a predefined budget. The game functions by monitoring time taken to complete various activities and through evaluating the correlation between actual progress and an expected normal path of completion.
Billis et al. [28] describe the use of "exergaming," a concept where games use exercise as a primary mode of interaction with the aim to slow cognitive degeneration. The authors have developed a system entitled the FitForAll that utilises the Nintendo Wii remote controller and balance board to recognise user motion and allow them to interact with a virtual environment.

Reference [28] utilised affective evaluation to gain feedback. They concluded that "FitForAll" imparted a positive feel for test participants, with the game itself being easy to use and helpful. Moreover affective evaluation was able to correctly predict $46.8 \%$ of usability scores for the test system used.

He et al. [29] have designed a serious $3 \mathrm{~d}$ gesture recognition game in an attempt to increase a participant's physical exercise, memory, reasoning, calculation, and spatial recognition in order to prevent dementia using a series of four games in each of those cognitive areas. Their developed system utilises a $3 \mathrm{~d}$ camera to recognise full body motion in a user, identifying specific actions made that are used to control and interact with the gaming environment.

Chilukoti et al. [30] have developed a system to improve physical exercise and cognitive training of Alzheimer's patients through implementing a stationary cycle alongside a multiple choice quiz game. The developed system focuses on assessing user's executive function including memory, judgement, matching, and problem solving capability.

De Urturi Breton et al. [2] have developed KiMentia, a system designed to enhance user memory and physical exercise using a Microsoft Xbox game and Kinect 3d sensor.

Their system consists of a number of different games that include constructing words by adding syllables to ones displayed, as well as correct classification and categorisation of words, for instance, correctly recognising that onion is a vegetable or medicine that can be found at a pharmacy. Their system uses graphical images for representation with physical movement needed to select options providing exercise.

MinWii [13] is a music therapy game that utilises an onscreen digitised piano keyboard that allows dementia patients to visualise the notes of common songs such as nursery rhymes being played in order to engage them to play along using a Wii remote.

Helping to increase cognitive abilities and build selfesteem in sufferers whilst providing an easy to use, engaging, and enjoyable social interface between fellow sufferers and their carers.

Tapus [31] has designed a system that couples the use of an assistive robot alongside a song discovery game. The robot is used to present tasks to the user in the form of song excerpts that the user must identify by pressing appropriate buttons. The robot provides a form of one to one social interaction and stimuli in order to heighten and exercise the player's conversational and mental skills.

Anguera et al. [32] have developed a $3 \mathrm{~d}$ car racing game that incorporates multitasking as a means to enhance cognitive control in users. Their game "NeuroRacer" is designed to simultaneously have players recognise and respond to onscreen signs whilst manoeuvring a vehicle along a winding road. 
The overall effectiveness of this training was said to enhance multitasking performance, short-term memory, and attention by $400 \%$ in older (60-85 year old) users where cognitive decline is most prevalent, to a level superior to 20year-old users, and retain this effect over a six-month period.

Ultimately the main purpose of the games reviewed in this section is to help promote mental and/or physical exercise in order to train and rehabilitate sufferers with some form of cognitive deficit. However, importantly, they are unable to offer any real predictive capability of the onset of mental illness that would be of clear importance to dementia care.

3.3. Predictive Mechanisms. Jimison et al. [33] have developed an interesting form of verbal fluency assessment that forms part of standard psychological testing to ascertain a range of mental illnesses such as dementia and Alzheimer's disease. Verbal fluency involves the patient associating known words to a selected specific letter or category. The devised system requires test subjects to reorganise a collection of letters into as many possible meaningful words and ultimately utilises heuristics, such as the complexity of words generated and speed of word generation to gauge overall test subject performance.

Kurata et al. [17] have devised a very simple set of tests that closely equate to the rated effectiveness of the Mini-Mental State Examination (MMSE) and the Revised Hasegawa Dementia Scale (HDS-R) for dementia assessment. Furthermore this [17] methodology is said to be able to assess whole brain function of patients through employing the use of four different types of gameplay that comprise of

(1) identifying similar cards in a random arrangement by flipping them sequentially,

(2) identifying the difference between two images,

(3) arranging four pictures in a correct specific order,

(4) differentiating between a sequence of images and marking them accordingly.

Shamsuddin et al. [18] have designed a series of virtual environments to gauge topographical disorientation in a user's ability to navigate space. The system is said to have a $90 \%$ degree of average effective accuracy of indication for MCI that is thought to be a precursor towards dementia, providing a significant level of prediction.

\section{Discussion}

The methodology outlined by Li et al. [20] is considerably less efficient and offers no real additional effectiveness in contrast to the standard paper based MoCA assessment that poses a lesser cognitive load in use and can be undertaken at reduced cost and in less time.

It is also incorrect to state that dementia can be prevented, as the authors text of [20] seems to suggest, simply by adopting and maintaining an active physical and mental state; although this has been proven to help reduce its effect, cognitive decline is still possible [34].

Imbeault et al. [22] state that Alzheimer's patients require specialist noninvasive and assistive training in the four key areas which are (i) memory, (ii) planning skills, (iii) initiative, and (iv) perseverance which their game accommodates. However, there are additional factors of equal importance that are absent from their design such as visuospatial awareness [18]. Furthermore, their mechanism itself offers no recognisable advantage over other conventionally available commercial releases such as Dr. Kawashima's brain training or Cooking Mama for the Nintendo DS that currently feature an adaptive difficulty level. The [22] system also suggests that it utilises a nonportable PC making it more expensive and cumbersome, restricting its potential.

Although the training technique used by Prokopenko et al. [23] is somewhat effective it is limited in terms of effectiveness, usability, and appeal. Other studies make better use of gaming technology available such as both the [18] and [17] environments. It is also felt that this system would be more efficient to develop and easier to use by adopting a single game level that requires only one game development toolkit and employs a largely uniform style of gameplay and mode of interaction. In contrast to a series of independent, dissimilar mechanisms and tasks that are presented to the user to the detriment of their ease of use and also to testing continuity.

The principal concerns regarding the system described by de la Guia [24] are in essence twofold. Firstly, superior rehabilitative technologies for cognitive impairment have been developed that provide greater function $[2,23]$. These alternatives simultaneously require significantly less learning and effort, making them easier to use than the aforementioned system. Secondly, many alternatives are a great deal less expensive to produce and implement yet still employ a computer such as [17]. As a result the system affords no real additional benefits.

The game developed in Lin and Chen's works [25] lacks ease of use and assumes that older people will have experience of planting orchids. It is an overly complex game that requires users to recognise key aspects of orchid planting such as temperature, humidity, luminance, and watering that are not easily apparent in a virtual environment.

Chang et al. [26] limit the use of their system to Chinese users alone and it is debatable as to how many people can still relate to food stamps from many years ago, in contrast to conventional Chinese currency that they will most likely have experience with. Although the touch screen interface has proven to be largely easy to use, more difficult games were hard to master by participants, highlighting the need for a significant level of training to be used by elderly patients effectively.

Although testing for Lopez-Martínez et al. [27] has ascertained a degree of similarity between the more conventional modified 6 elements test $(6 \mathrm{E})$ and dysexecutive function tests undertaken in parallel with the game, its efficacy remains inconclusive. Furthermore, the level of benefit in this game for users is extremely limited in contrast to alternatives such as that developed by [17]. There is also a large amount of cognitive complexity in the user interface at nearly every stage of the game with significant amounts of text. These issues combined limit the usability of the game, particularly for those with dementia. 
With reference to Billis et al. [28] a number of studies such as that of [10] have concluded that physical and social activities can delay cognitive decline in particular when used in conjunction with cognitive activities. Yet this very same study goes on to state that Wii sports type derivatives can cause usability problems for dementia sufferers as a result of the complexity represented by the controller.

However, the system used is also restrictive in terms of the low level of portability of the system. This means that it is limited to domestic use alone. Additionally, participants with cognitive dysfunction will require extensive training and supervision. There is also considerable cost that is incurred for the initial purchase of the gaming hardware needed which is over and above a typical computer, such as the balance board.

Furthermore, the developed game is greatly overcomplicated using multiple control mechanisms such as the potentially tricky to use balance board. Easier to use gesture recognition technology such as Xbox Kinect has already been utilised to some extent, for similar purposes in existing commercially available titles such as Dr. Kawashima's body and brain exercise for the Xbox 360 [35]. Additionally, the system may run into difficulty if the participants are older patients with dementia as the interface is complex. This is further compounded by the fact that physical exertion can cause discomfort and trigger agitation in a user with dementia, which increases the risk of resentment to the extent it may even prevent the actual use of the system [36].

The system outlined by Chilukoti et al. [30] lacks the ability to test additional key areas of cognitive function such as retaining motivation or assessing performance of daily activities $[22,27]$. As such this fails to provide a complete user diagnostic assessment mechanism comparable to mental test batteries currently utilised.

This [30] mechanism also poses a safety risk to potential users, particularly as the system will be difficult to use independently. Furthermore, the authors themselves state that there needs to be a device to stop users from overworking themselves.

It can be safely ascertained that the developed system is overly cumbersome, highly invasive, and more expensive than alternative systems discussed and requires a greater degree of supervision, rendering it both ineffective and inefficient in contrast to most alternative techniques presented in this review, negating its use.

The major advantage of using the Kinect platform, and as stated by the developers of Kimentia [2], is that it provides potentially the easiest form of game manipulation without having to use a controller. However, in contrast to the article, it is evident that users with severe muscle problems that would find controller buttons difficult to press (identified as potential candidates for their new system) will have issues standing and making physical movements for the extended periods of time that this game may require.

Moreover, the Kinect camera set up requires a high degree of user calibration to correctly recognise user movements. This can be a quite lengthy process that limits its independent use for people with dementia.
Kimentia [2] simply concentrates on the language development centres of the brain and is therefore not as effective as its counterparts, such as the [17] system that provides a more comprehensive potential rehabilitative capacity for cognitive impairment, and one that requires less training.

Although a high degree of users were able to complete the task set reasonably quickly for the MinWii system [13] the authors themselves discovered that in actual use MinWii requires a great deal of assistance limiting its use for dementia patients on their own, primarily due to initial training required. This is further hampered by the technical complexity of having to install and set up the required hardware and the initial cost of having to purchase it, in contrast to using a PC that they may already have.

Furthermore, the analysis undertaken by [13] of user behaviour, expressions, wiimote use and a number of assistive interventions, (such as interjecting with verbal cues and actual physical assistance required) that simply relies on video recordings observed after the test. In contrast to noting these during actual testing, is likely to have missed a number of potentially important events and as a result can have an impact on the overall accuracy of findings.

The song identification game as developed by Tapus [31] is a valid basic concept to train memory, organisation, and planning skills in users, but to the detriment of other skills such as daily living activities or spatial recognition that are needed in order to provide a more comprehensive assessment mechanism.

Music was found to be an effective stimulus to raise interest, motivation, and responsiveness in test subjects. This worked in conjunction with a robot capable of autonomously adaptive behaviour that was able to successfully improve task performance and attention over time. However, the use of a robot lacks efficiency in terms of cost and complexity. It is also felt that maintaining cognitive attention of a user can be achieved comparatively more effectively through using a conventional interactive game environment alone as demonstrated by [18].

Unfortunately, this comparison to 20 year olds as used by Anguera et al. [32] is flawed due to the fact that the older users in the study have extensively played the game over a considerable period of time whilst the younger player group in question was completely new to the game, posing a significant disadvantage.

Neuroracer only utilises interference resolution and multitasking capability in assessing test subject performance as a means to rehabilitate users. Although it shows potential, it lacks focus on some cognitive functions such as reasoning, static orientation, and abstraction.

Neuroracer is also only capable of exercising the frontal brain region alone whereas alternative systems reviewed such as that of $[17,23]$ are each able to provide a much more comprehensive level of brain training in conjunction with some level of multitasking for the player.

Furthermore, the Anguera mechanism [32] provides no information as to the effects of cognitive multitasking in relation to users having dementia or MCI. As a result it provides no usable or feasible comparison to the alternative systems reviewed, ultimately rendering its usefulness in application questionable. 
The Kurata [17] technique in particular is able to provide a potential rehabilitation mechanism alongside an effective proven prediction and assessment function.

The study undertaken by Jimison et al. [19] has a serious limitation in that user strategies formed to complete a game may be very different from the shortest possible number of moves for its completion influencing recorded performance and effectively rendering the data gleaned ineffectiv and unreliable.

The authors acknowledge key indicators of cognitive impairment such as attention and memory as absent from their devised performance metric, yet they are used for the more general psychological assessment undertaken. As such the testing methodology is restricted to a finite set of parameters that does not provide a conclusive assessment or comparability to any known diagnostic technique.

In operation the Jimison et al. [33] system has been found to provide a similar level of effectiveness to conventional cognitive assessment techniques in the area of verbal fluency and is said to be able to predict scores on the standard neuropsychological assessment. However, verbal fluency itself represents only a small part of the neuropsychological assessment spectrum and in itself does not provide any conclusive or comprehensive diagnosis of dementia, particularly not its prediction.

The system as developed by Kurata et al. [17] is somewhat invasive as the user is able to recognise the testing process and although the methodology is able to provide effective early diagnosis of dementia, it is not able to predict the disease prior to a patient becoming ill and presenting clear executive deficit. It would also be interesting to incorporate a number of other assessments such as verbal fluency alongside the visual tests used in a single game level and observe any additional benefit and resemblance to results from the best of conventional Alzheimer/dementia evaluation methods currently available.

The mechanism developed by Shamsuddin et al. [18] is not able to predict the onset of dementia prior to the test subject becoming ill with MCI, nor does it offer the same degree of comprehensive testing that a conventional paper based test can afford.

The core element of this system that requires a user to navigate a virtual environment can easily be replicated using a standard game development platform such as Unreal, where it may be coupled with digital versions of other standardised mental health tests to provide much more substantial testing potential.

Furthermore, a single continuous level can be developed to provide more comprehensive results, increase quality of data acquisition in contrast to a number of separate games, and simplify both development effort and ease of use for the test platform.

It can be ascertained from the literature reviewed that there are clearly a large number of interactive computer game based mechanisms orientated towards dementia sufferers.

However these are primarily rehabilitative mechanisms that attempt to enhance the capability of a sufferer's executive function and are rarely capable of any predictive capacity, particularly in terms of affording diagnosis prior to the onset of dementia.

In terms of prediction for dementia there are a very limited number of games with each simply relying on providing a simple diagnostic assessment for a previous stage of the diseases lifecycle such as at MCI or early dementia.

Although there is a degree of validity in terming these mechanisms as predictive, they are to a high degree unintelligent offering no real innovation to current testing methodologies employed such as established mental assessment batteries. It would also be far more useful to predict cognitive impairment at a stage where it could perhaps be successfully mitigated without any treatment.

Interestingly the systems with higher functional success rates adopt a disparate series of different gaming mechanisms in the name of providing a comprehensive test. However, each individual mechanism involves substantially different gameplay by the user to function correctly as intended $[17,18]$. This negatively impacts ease of use and learnability that needs to be as close to effortless as possible for a person with dementia to use effectively.

Although some mechanisms such as [17] state they can provide a similar level of testing efficacy to standard mental assessment batteries such as the MMSE, they fail to provide the exact level of testing rigour or comprehensiveness of such a mechanism. In fact both the MMSE and the naturalistic action test can be comfortably intertwined and digitised in a single $3 \mathrm{~d}$ game level using a conventional game engine such as Unreal.

Also the vast majority of games reviewed are considerably invasive and require extensive supervision highly negating the possibility that they could ever be used by the patient regularly on their own or indeed provide the level of motivation needed in order for this to occur. The only exception being [19] that instead utilises a metric that is far too basic and fails to provide a comprehensive form of testing that is similarly effective to that of a contemporary neuropsychological test such as the MoCA.

Additionally some authors have implemented technologies that have a number of innate applications that seem to have gone unnoticed [17]. For instance, failing to recognise that the touchscreen interface used could easily be deployed using smart phone devices as a natural development and therefore increasing access to healthcare for a wide ranging audience using a readily available and already exploited platform.

Lumosity [14] is an example of a current online game that can be played on mobile devices and attempts to increase cognitive function through providing effective brain training exercises. However its long-term benefits particularly on memory are unproven. Furthermore Lumosity does not utilise the capabilities of current smartphone technology to provide any additional benefit to the user, highly refuting its efficacy for use.

If we consider the almost ubiquitous availability, use, and indeed ever expanding power and capabilities [37, 38] of modern mobile smartphones, we are presented with a device with a capability that by far exceeds its main purpose of simply making telephone calls and provides a logical exploration platform for dementia healthcare. 


\section{Conclusion}

No reviewed technique provides any actual insight into the accuracy of assessments made over the long term to gauge actual effectiveness in successfully diagnosing dementia prior to its onset. Moreover no assessment technique offers any additional tangible benefit over the standard paper based techniques currently utilised, nor does it offer a comparably comprehensive range of assessment and as such calls into question the validity of deploying serious games as a genuine useful alternative.

Additionally, there is little information as to the usefulness of any rehabilitative mechanisms found to be effective in testing in terms of real life long-term positive impact beyond a six-month period.

The key reasons for the ineffectiveness of the game mechanisms reviewed in contrast to conventional paper based methods are firstly that they mainly require a substantial amount of time to use and acquire usable data from, this can range up to a year with [33] in sharp contrast to the few minutes needed for a test such as the MoCA. Further complicated by the need for certain games to structure this time such as [32], that requires an hour of independent use, three times a week for a month. This length of play can be extremely arduous in itself for dementia sufferers without the added difficulty of having to adhere to a specific schedule without supervision. Conversely, mechanisms reviewed that require less time to implement such as $[2,13,31]$ suffer from a significant level of complexity and intrusiveness, again needing supervision and even specialist hardware that can be costly for those on lower incomes and difficult to set up and use by a lone individual with cognitive deficit, therefore further supporting grounds for their unsuitability.

Currently for the purpose of predicting dementia there is no mobile phone native system in use or available and therefore this provides a compelling research pursuit. Particularly given the fact that mobile games can be used independently and effectively by dementia sufferers [10] as demonstrated by the game Lumosity [14].

This is further reinforced by the work of $[39,40]$ that have glimpsed the potential use of harnessing smartphone technologies for assessment and prediction of mental illness at a treatable stage prior to dementia or even MCI using native sensing technology found in nearly all smartphones. However, their works are too basic and fail to provide any tangible comparison in terms of effectiveness that can favourably parallel traditional mental assessment batteries currently in practice.

There is also a growing level of concern regarding digital dementia, where marked cognitive decline on par with serious psychological illness or head injury is observed amongst users of digital technology and computer games, having a serious effect on brain development [41]. By utilising an in game assessment mechanism as proposed would provide an ideal, effective and nonintrusive means to investigative this.

The authors of this review anticipate to build upon a combination of the basic premises as outlined by $[17,18,39,40]$ and attempt to produce a mobile phone based $3 \mathrm{~d}$ virtual interactive environment monitoring user response times and behaviour. In order to provide a noninvasive medical grade cognitive impairment predictive capability does not simply closely approximate but actually exceeds both the Montreal Cognitive Assessment (MoCA) scale and Mini-Mental State Examination (MMSE).

\section{Conflict of Interests}

The authors declare that there is no conflict of interests regarding the publication of this paper.

\section{References}

[1] A. Singh-Manoux and M. Kivimäki, "The importance of cognitive ageing for understanding dementia," Age, vol. 32, no. 4, pp. 509-512, 2010.

[2] Z. S. de Urturi Breton, B. G. Zapirain, and A. M. Zorrilla, "KiMentia: kinect based tool to help cognitive stimulation for individuals with dementia," in Proceedings of the IEEE 14th International Conference on e-Health Networking, Applications and Services (Healthcom '12), pp. 325-328, 2012.

[3] C. Shanley, "Promoting advance care planning to reduce the impact of dementia: a new web resource from Alzheimer's Australia," BMJ Supportive \& Palliative Care, vol. 3, no. 2, pp. 237-237, 2013.

[4] World Health Organization, Dementia: A Public Health Priority, World Health Organization and Alzheimer's Disease International, 2012, http://www.alz.co.uk/WHO-dementia-report.

[5] Alzheimers Society, 2013, http://alzheimers.org.uk/site/scripts/ documents_info.php?documentID=2164.

[6] L. Waite, "Dementia: an international crisis?" Perspectives in Public Health, vol. 132, no. 4, pp. 154-155, 2012.

[7] R. Luengo-Fernandez, J. Leal, and A. M. Gray, "UK research expenditure on dementia, heart disease, stroke and cancer: are levels of spending related to disease burden?" European Journal of Neurology, vol. 19, no. 1, pp. 149-154, 2012.

[8] United Nations, Ageing in the Twenty-First Century: A Celebration and $a$ Challenge, UNFPA and HelpAge International, 2012, http://unfpa.org/ageingreport/.

[9] R. C. Petersen, R. Doody, A. Kurz et al., "Current concepts in mild cognitive impairment," Archives of Neurology, vol. 58, no. 12, pp. 1985-1992, 2001.

[10] S. McCallum and C. Boletsis, "A taxonomy of serious games for dementia," in Games for Health, pp. 219-232, Springer Fachmedien, Wiesbaden, Germany, 2013.

[11] S. McCallum and C. Boletsis, "Dementia games: a literature review of dementia-related serious games," in Serious Games Development and Applications, vol. 8101 of Lecture Notes in Computer Science, pp. 15-27, Springer, Berlin, Germany, 2013.

[12] K. P. Padala, P. R. Padala, T. R. Malloy et al., "Wii-fit for improving gait and balance in an assisted living facility: a pilot study," Journal of Aging Research, vol. 2012, Article ID 597573, 6 pages, 2012.

[13] M. Boulay, S. Benveniste, S. Boespflug, P. Jouvelot, and A.-S. Rigaud, "A pilot usability study of MINWii, a music therapy game for demented patients," Technology and Health Care, vol. 19, no. 4, pp. 233-246, 2011.

[14] M. Finn and S. McDonald, "Computerised cognitive training for older persons with mild cognitive impairment: a pilot study using a randomised controlled trial design," Brain Impairment, vol. 12, no. 3, pp. 187-199, 2011. 
[15] B. Fernández-Calvo, R. Rodríguez-Pérez, I. Contador, A. Rubio-Santorum, and F. Ramos, "Efficacy of cognitive training programs based on new software technologies in patients with Alzheimer-type dementia," Psicothema, vol. 23, no. 1, pp. 44-50, 2011.

[16] H. Tobiasson, Physical Action Gaming and Fun as a Tool within Elderly Care: Game Over or Play It Again and Again, IEA, Beijing, China, 2009.

[17] T. Kurata, Y. Omote, M. Ikeda et al., "The usefulness of a simple computerized touch panel-type screening test for Alzheimer's disease patients," in Proceedings of the 6th International Conference on Complex Medical Engineering (CME '12), pp. 215-217, July 2012.

[18] S. N. W. Shamsuddin, H. Ugail, V. Lesk, and E. Walters, "VREAD: a virtual simulation to investigate cognitive function in the elderly," in Proceedings of the International Conference on Cyberworlds (CW '12), pp. 215-220, Darmstadt, Germany, September 2012.

[19] H. Jimison, M. Pavel, J. McKanna, and J. Pavel, "Unobtrusive monitoring of computer interactions to detect cognitive status in elders," IEEE Transactions on Information Technology in Biomedicine, vol. 8, no. 3, pp. 248-252, 2004.

[20] H. Li, T. Zhang, T.-C. Yu, C.-C. Lin, and A. M. K. Wong, "Combine wireless sensor network and multimedia technologies for cognitive function assessment," in Proceedings of the $3 \mathrm{rd}$ International Conference on Intelligent Control and Information Processing (ICICIP '12), pp. 717-720, July 2012.

[21] C. Jacova, A. Kertesz, M. Blair, J. D. Fisk, and H. H. Feldman, "Neuropsychological testing and assessment for dementia," Alzheimer's \& Dementia, vol. 3, no. 4, pp. 299-317, 2007.

[22] F. Imbeault, B. Bouchard, and A. Bouzouane, "Serious games in cognitive training for Alzheimer's patients," in Proceedings of the IEEE 1st International Conference on Serious Games and Applications for Health (SeGAH '11), pp. 1-8, November 2011.

[23] S. V. Prokopenko, E. Y. Mozheyko, T. D. Koryagina et al., "Neurorehabilitation of poststroke cognitive impairments with the use of computed programs," in Proceedings of the International Conference on Virtual Rehabilitation (ICVR '11), pp. 1-6, June 2011.

[24] E. de la Guia, M. D. Lozano, and V. R. Penichet, "Cognitive rehabilitation based on collaborative and tangible computer games," in Proceedings of the 7th International Pervasive Computing Technologies for Healthcare (PervasiveHealth '13), pp. 389-392, Venice, Italy, May 2013.

[25] M.-H. Lin and S.-H. Chen, "Key factors influencing the development of orange technology game: an exploratory study on orchid planting game," in Proceedings of the PICMET '12: Technology Management for Emerging Technologies (PICMET '12), pp. 1463-1470, Vancouver, Canada, July-August 2012.

[26] K. Chang, N. An, J. Qi et al., "Food stamps: a reminiscence therapy tablet game for Chinese seniors," in Proceedings of the ICME International Conference on Complex Medical Engineering (CME '13), pp. 556-561, Beijing, China, May 2013.

[27] A. Lopez-Martínez, S. Santiago-Ramajo, A. Caracuel, C. VallsSerrano, M. J. Hornos, and M. J. Rodríguez-Fortiz, "Game of gifts purchase: Computer-based training of executive functions for the elderly," in Proceedings of the IEEE 1st International Conference on Serious Games and Applications for Health (SeGAH '11), pp. 1-8, November 2011.

[28] A. S. Billis, E. I. Konstantinidis, A. I. Ladas, M. N. Tsolaki, C. Pappas, and P. D. Bamidis, "Evaluating affective usability experiences of an exergaming platform for seniors," in Proceedings of the 10th International Workshop on Biomedical Engineering, pp. 1-4, Kos, Greece, October 2011.

[29] G. F. He, J. W. Park, S. K. Kang, and S. T. Jung, "Development of gesture recognition-based serious games," in Proceedings of the IEEE-EMBS International Conference on Biomedical and Health Informatics (BHI '12), pp. 922-925, January 2012.

[30] N. Chilukoti, K. Early, S. Sandhu, C. Riley-Doucet, and D. Debnath, "Assistive technology for promoting physical and mental exercise to delay progression of cognitive degeneration in patients with dementia," in Proceedings of the IEEE Biomedical Circuits and Systems Conference Healthcare Technology (BiOCAS '07), pp. 235-238, Montreal, Canada, November 2007.

[31] A. Tapus, "Improving the quality of life of people with dementia through the use of socially assistive robots," in Proceedings of the Advanced Technologies for Enhanced Quality of Life (AT-EQUAL '09), pp. 81-86, July 2009.

[32] J. A. Anguera, J. Boccanfuso, J. L. Rintoul et al., "Video game training enhances cognitive control in older adults," Nature, vol. 501, no. 7465, pp. 97-101, 2013.

[33] H. Jimison, M. Pavel, and T. Le, "Home-based cognitive monitoring using embedded measures of verbal fluency in a computer word game," in Proceedings of the 30th Annual International Conference of the IEEE Engineering in Medicine and Biology Society (EMBS '08), pp. 3312-3315, Vancouver, Canada, August 2008.

[34] S. Gauthier, B. Reisberg, M. Zaudig et al., "Mild cognitive impairment," The Lancet, vol. 367, no. 9518, pp. 1262-1270, 2006.

[35] Microsoft, Dr. Kawashima, 2011, http://marketplace.xbox.com/ en-GB/Product/Dr-Kawashimas-Body-and-Brain-Exercises/ 66acd000-77fe-1000-9115-d8024e4d0827.

[36] H. Ragneskog, "Probable reasons for expressed agitation in persons with dementia," Clinical Nursing Research, vol. 7, no. 2, pp. 189-206, 1998.

[37] S. Yang, S. Kurnia, and S. P. Smith, "The impact of mobile phone use on individual social capital," in Proceedings of the 44th Hawaii International Conference on System Sciences (HICSS '10), Kauai, Hawaii, USA, January 2011.

[38] C. A. Opperman and G. P. Hancke, "Smartphones as a platform for advanced measurement and processing," in Proceedings of the IEEE International Instrumentation and Measurement Technology Conference (I2MTC '12), pp. 703-706, Graz, Austria, May 2012.

[39] M. Sung and A. Pentland, Minimally-Invasive Physiological Sensing for Human-Aware Interfaces, HCI International, 2005.

[40] A. Brahms, Representation Error for Real Numbers in Binary Computer Arithmetic, Paper R, IEEE Computer Group Repository, 2005.

[41] G. W. Small, P. Siddarth, L. M. Ercoli, S. T. Chen, D. A. Merrill, and F. Torres-Gil, "Healthy behavior and memory self-reports in young, middle-aged, and older adults," International Psychogeriatrics, vol. 25, no. 6, pp. 981-989, 2013. 

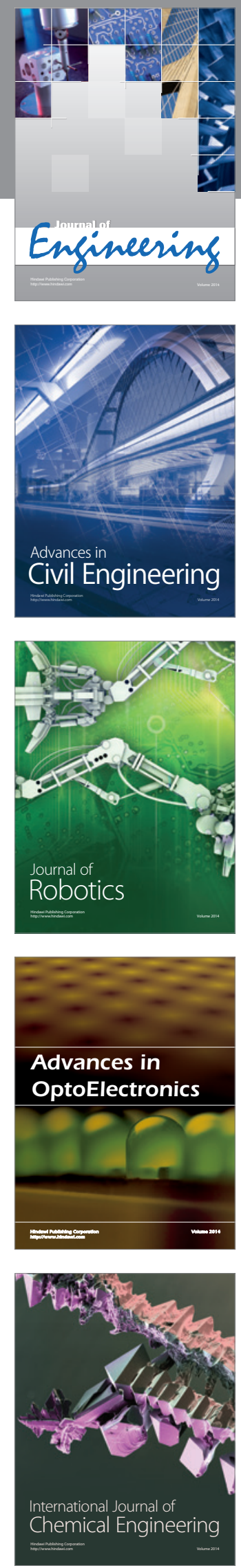

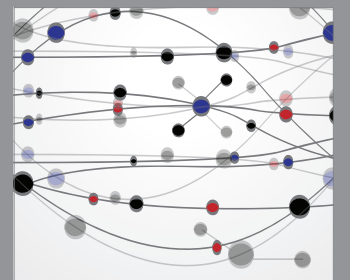

The Scientific World Journal
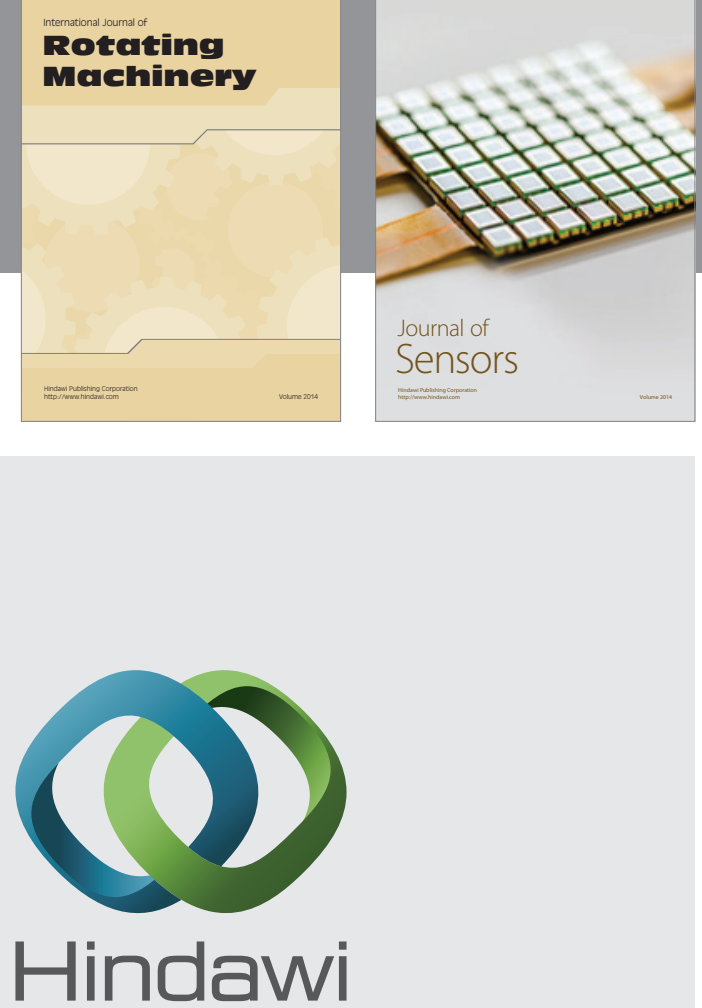

Submit your manuscripts at http://www.hindawi.com
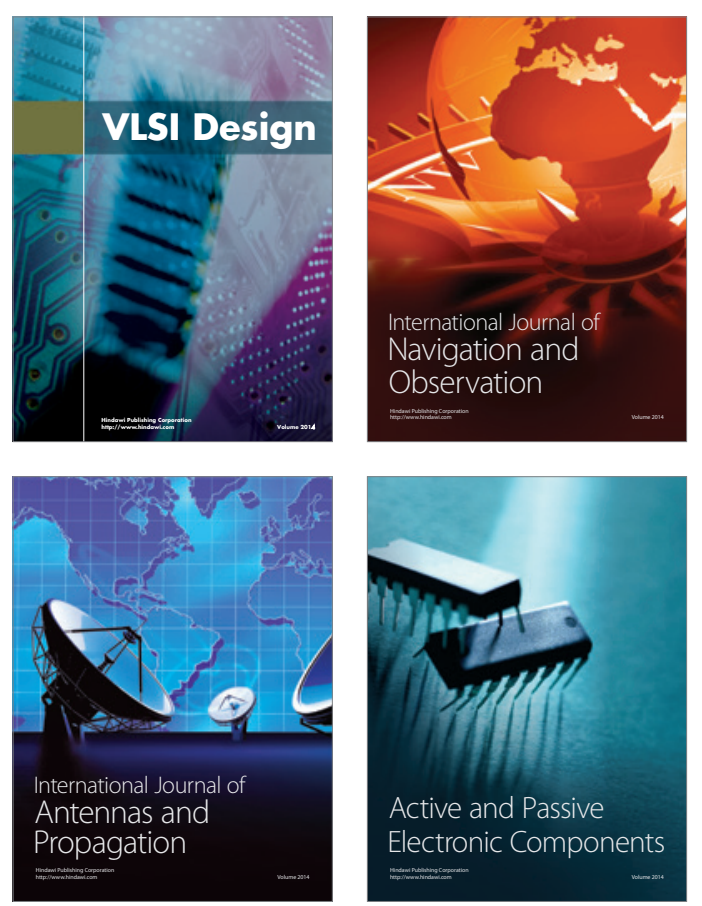
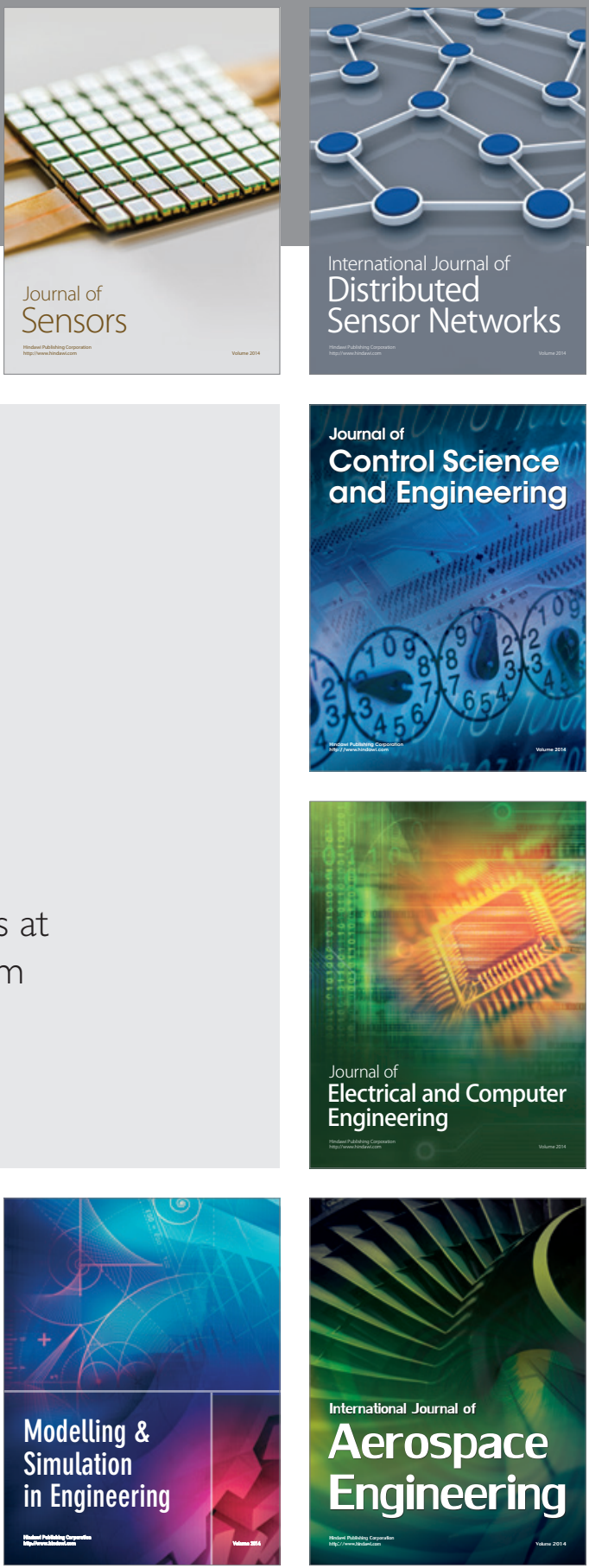

Journal of

Control Science

and Engineering
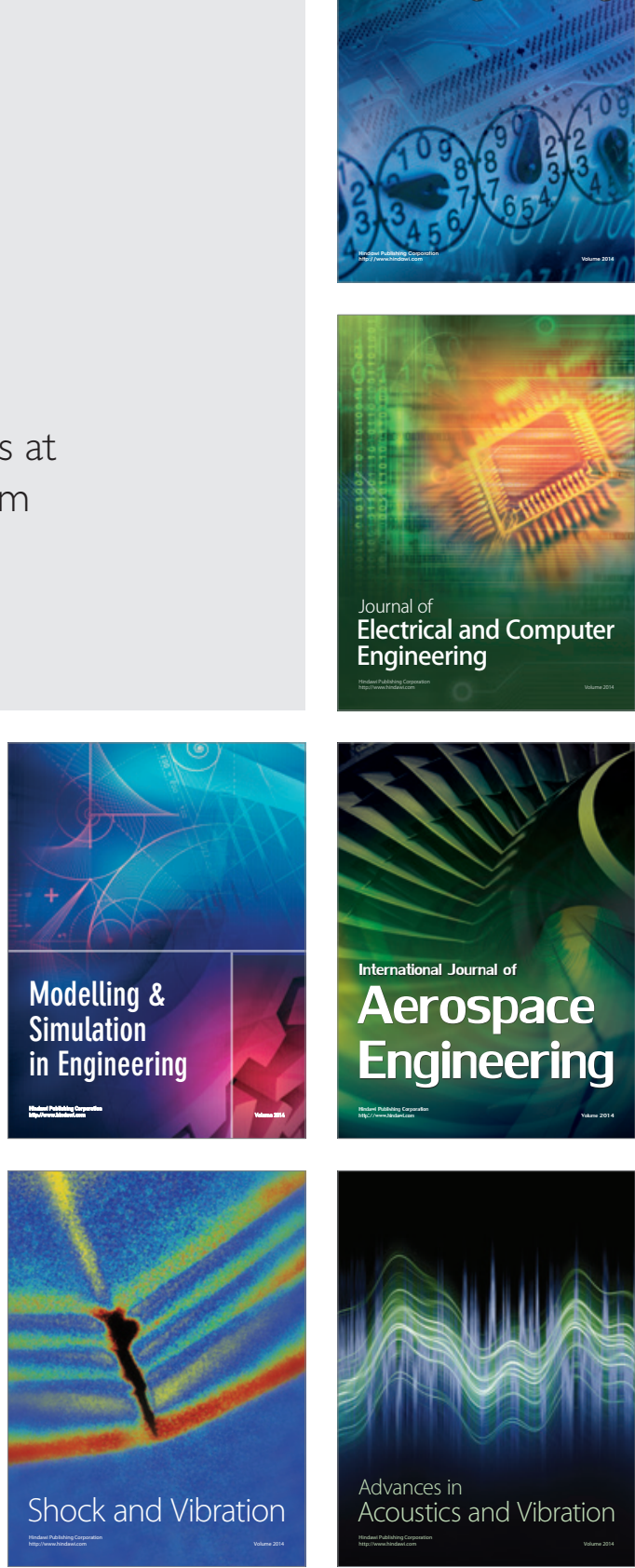